\title{
Análise dos níveis de carga proviral em portadores de HTLV-1 com diferentes condições de comprometimento neurológico
}

\author{
Analysis of proviral load levels in HTLV-1 carriers with different neurological impairment \\ conditions
}
Análisis de los niveles de carga proviral en portadores de HTLV-1 con diferentes condiciones de deterioro neurológico

Izabela Mendonca de Assis ORCID: https://orcid.org/0000-0002-9757-6799 Universidade Federal do Pará, Brasil

E-mail: izabela_stm@hotmail.com

Mariângela Moreno Domingues ORCID: https://orcid.org/0000-0001-8787-7101 Universidade Federal do Pará, Brasil

E-mail: mmorenodomingues@gmail.com

Danilo de Souza Almeida ORCID: https://orcid.org/0000-0003-4528-9269 Universidade Federal do Pará, Brasil

E-mail: almeida.danilo.ufpa@gmail.com

Louise de Souza Canto Covre ORCID: https://orcid.org/0000-0002-7510-6147 Universidade Federal do Pará, Brasil E-mail: louisecanto12@yahoo.com.br

Debora Glenda Lima de La-Roque ORCID: https://orcid.org/0000-0001-9032-7485 Universidade Federal do Pará, Brasil E-mail: debora.laroque@gmail.com

Ingrid Christiane Silva

ORCID: https://orcid.org/0000-0002-9753-6041 Universidade Federal do Pará, Brasil E-mail: ingridchristiane22@gmail.com

Akim Felipe Santos Nobre ORCID: https://orcid.org/0000-0002-8501-9346 Universidade Federal do Pará, Brasil E-mail: akimnobre@hotmail.com

Mariza da Silva Borges

ORCID: https://orcid.org/0000-0003-2942-423X Universidade Federal do Pará, Brasil E-mail: marizaborges90@ @otmail.com

Cássia Cristine Costa Pereira ORCID: https://orcid.org/0000-0002-3175-1742 Universidade Federal do Pará, Brasil E-mail: cassiaccpereira@gmail.com

Barbara Brasil Santana ORCID: https://orcid.org/0000-0002-5621-9701 Universidade Federal do Pará, Brasil

E-mail: biomedicinabarbara@gmail.com

Antonio Carlos Rosario Vallinoto ORCID: https://orcid.org/0000-0003-1135-6507 Universidade Federal do Pará, Brasil E-mail: vallinoto@ufpa.br

Maísa Silva de Sousa

ORCID: https://orcid.org/0000-0001-9944-7838 Universidade Federal do Pará, Brasil E-mail: maisasousa@ufpa.br

\section{Resumo}

Objetivos: Analisar os níveis da carga proviral (CPV) em portadores de HTLV-1 com diferentes condições de comprometimento neurológico. Metodologia: Estudo transversal, realizado de março a outubro de 2017, com 43 
pacientes com HTLV-1, divididos em três grupos por ordem decrescente de envolvimento neurológico, segundo proposta atualizada dos critérios de diagnóstico clínico para HAM/TSP: Definidos para HAM/TSP (Grupo 1, n=7); Prováveis/Possíveis para HAM/TSP (Grupo 2, n=9); Sem HAM/TSP (Grupo 3, n=27). O teste ANOVA (um critério) foi utilizado para verificar a diferença entre as médias de CPV dos grupos (p-valor $\leq 0,05)$. Resultados: Os grupos

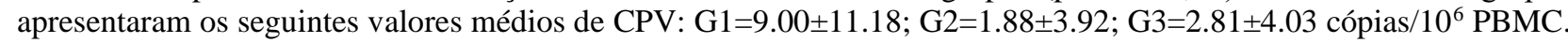
Na comparação intergrupo, da diferença entre as médias de CPV, foi observada diferença significativa entre os grupos 1 e 2 e entre os grupos 1 e 3 ( $p<0,05)$; não foi observada uma média significativamente maior de CPV nos grupos de infectados pelo HTLV-1 com comprometimento neurológico, frente aos infectados sem comprometimento neurológico ( $\mathrm{p}=0,7063)$. Conclusão: A CPV em portadores de HTLV-1 parece auxiliar a avaliação clínica do comprometimento neurológico na classificação dos casos clássicos de HAM/TSP, mas não se mostra um indicador importante de condições clínicas iniciais da HAM/TSP.

Palavras-chave: HTLV-1; Paraparesia espástica tropical; Distúrbios neurológicos; Carga proviral.

\begin{abstract}
Objectives: To analyze the levels of proviral load (CPV) in patients with HTLV-1 with different conditions of neurological impairment. Methodology: Cross-sectional study, conducted from March to October 2017, with 43 patients with HTLV-1, divided into three groups in decreasing order of neurological involvement, according to the updated proposal of the clinical diagnosis criteria for HAM / TSP: Defined for HAM / TSP (Group 1, $\mathrm{n}=7$ ); Probable / Possible for HAM / TSP (Group 2, $\mathrm{n}=9$ ); Without HAM / TSP (Group 3, $\mathrm{n}=27$ ). The ANOVA test (one criterion) was used to verify the difference between the groups' mean CPV (p-value $\leq 0.05$ ). Results: The groups presented the following average CPV values: $\mathrm{G} 1=9.00 \pm 11.18 ; \mathrm{G} 2=1.88 \pm 3.92 ; \mathrm{G} 3=2.81 \pm 4.03$ copies $/ 10^{6} \mathrm{PBMC}$. In the intergroup comparison, of the difference between the means of CPV, a significant difference was observed between groups 1 and 2 and between groups 1 and 3 (p <0.05); a significantly higher mean of CPV was not observed in the HTLV-1 infected groups with neurological impairment, compared to those infected without neurological impairment ( $p=0.7063$ ). Conclusion: CPV in patients with HTLV-1 seems to help the clinical evaluation of neurological impairment in the classification of classic cases of HAM/TSP, but it is not an important indicator of initial clinical conditions of HAM/TSP.
\end{abstract}

Keywords: HTLV-1; Tropical spastic paraparesis; Neurological disorders; Proviral load.

\title{
Resumen
}

Objetivos: Analizar los niveles de carga proviral (CPV) en pacientes con HTLV-1 con diferentes condiciones de deterioro neurológico. Metodología: Estudio transversal, realizado de marzo a octubre de 2017, con 43 pacientes con HTLV-1, divididos en tres grupos en orden decreciente de afectación neurológica, de acuerdo con la propuesta actualizada de los criterios de diagnóstico clínico para HAM/TSP: Definido para HAM/TSP (Grupo 1, n = 7); Probable / Posible para HAM/TSP (Grupo 2, $n=9$ ); Sin HAM/TSP (Grupo 3, n = 27). Se utilizó la prueba ANOVA (un criterio) para verificar la diferencia entre el CPV medio de los grupos (valor $\mathrm{p} \leq 0,05$ ). Resultados: Los grupos presentaron los siguientes valores promedio de CPV: G1 = 9,00 $\pm 11,18 ; \mathrm{G} 2=1,88 \pm 3,92 ; \mathrm{G} 3=2,81 \pm 4,03$ copias $/ 10^{6}$ PBMC. En la comparación intergrupal, de la diferencia entre las medias de CPV, se observó una diferencia significativa entre los grupos 1 y 2 y entre los grupos 1 y 3 (p <0,05); no se observó una media significativamente mayor de CPV en los grupos infectados por HTLV-1 con deterioro neurológico, en comparación con los infectados sin deterioro neurológico ( $\mathrm{p}=0,7063$ ). Conclusión: la CPV en pacientes con HTLV-1 parece ayudar a la evaluación clínica del deterioro neurológico en la clasificación de casos clásicos de HAM/TSP, pero no es un indicador importante de las condiciones clínicas iniciales de HAM/TSP.

Palabras clave: HTLV-1; Paraparesia espástica tropical; Desórdenes neurológicos; Carga proviral.

\section{Introdução}

Os retrovírus são agentes importantes na morbimortalidade humana e têm provocado a emergência de diversas doenças nas últimas décadas. Entre estes, o Vírus T-linfotrópico Humano 1 (HTLV-1) têm persistência silenciosa e de longa duração no hospedeiro. Estima-se que mundialmente 15 a 20 milhões de pessoas estejam infectadas pelo HTLV-1 (The \& Bomford, 1993; Edlich, et al., 2003; Cook, et al., 2013), e o Brasil abrange cerca de dois milhões de pessoas desta população (Proietti, et al., 2005; Gessain \& Cassar, 2012).

Na Amazônia, a alta prevalência desta infecção tem tido destaque no cenário nacional, sendo o Pará o terceiro estado em número de casos entre doadores de sangue do Brasil (Carneiro-Proietti, et al., 2002; Catalan-Soares et al., 2005), o que tem suscitado estudos soro epidemiológicos e moleculares direcionados a comunidades com populações específicas: em Tomé-Açú (PA) com prevalência de 1,8\% para o HTLV-1 em 20 imigrantes japoneses residentes (Vallinoto, et al., 2004), na Ilha do 
Marajó (PA) com variação de zero a 2,06\% em comunidades remanescentes de quilombolas (Vallinoto, et al., 2006) e, em comunidades ribeirinhas de fácil acesso à Belém (PA) por meio de um estudo de base populacional rural foi encontrada uma prevalência de 1,14\% (Ferreira, et al., 2010).

Em estudos mais recentes, como de Da Costa (2013) e Silva (2018), foi encontrada uma alta e moderada endemicidade da infecção por HTLV em agregados familiares e população da área metropolitana de Belém, respectivamente. Em outro estudo, realizado com pessoas que usam drogas ilícitas no estado paraense, foi observada a prevalência de $3 \%$ para HTLV-1 (Oliveira-Filho, et al., 2019).

Dentre as doenças associadas à infecção pelo HTLV-1, destacam-se a Leucemia/Linfoma de Células T do Adulto (Adult T-Cell Leukemia/Lymphoma - ATL) e a Paraparesia Espástica Tropical/Mielopatia Associada ao HTLV-1 (HTLV-IAssociated Myelopathy or Tropical Spastic Paraparesis - HAM/TSP). Além destas, outras também têm sido descritas, como uveíte, dermatite infecciosa, polimiosite, neuropatias, etc (Araújo, 2019). Embora na maioria dos casos os portadores permaneçam assintomáticos, 2-6\% dos infectados desenvolvem ATL (Koga, et al., 2010; Kondo, et al., 1989) e outros 5\% desenvolvem HAM/TSP (Kaplan et al., 1990; Maloney et al., 1998). Acredita-se que desta porcentagem, os portadores que irão desenvolver HAM/TSP após a fase assintomática normalmente seja superior a 30 anos (Carod-Artal, 2009). Esta alteração neurológica degenerativa crônica é considerada uma das desordens mais comuns nos indivíduos infectados pelo HTLV-1, na qual o portador apresenta importante comprometimento das funções motoras, sensoriais e autonômicas (De Castro-Costa, et al., 2006; Osame, et al., 1990).

Em uma meta-análise que verificou a associação do HTLV-1 aos efeitos adversos à saúde, constatou-se uma gama de doenças estudadas em associação ao vírus, porém, o risco elevado de morte entre pessoas com HTLV-1 não é explicado pelos estudos disponíveis de morbidade. Assim, o ônus da doença associado ao vírus pode ser mais amplo do que o geralmente reconhecido (Schierhout, et al., 2020).

Para Araujo e Wedemann (2019), outras síndromes neurológicas podem estar associadas à HAM/TSP ou ocorrer isoladamente no indivíduo infectado pelo HTLV-1. Embora esse fato tenha sido largamente descrito ao longo dos anos, foi um pouco negligenciado pela literatura, que tem focado amplamente no HAM/TSP. Disfunção cognitiva, encefalopatia, bexiga neurogênica, doença do neurônio motor, miopatias inflamatórias, polineuropatia e disautonomia também podem ocorrer no paciente infectado pelo HTLV-1 e podem passar despercebidas a um médico pouco experiente.

A carga proviral (CPV) de HTLV-1 em células mononucleares do sangue periférico (peripheral blood mononuclear cells - PBMCs) é proposta como um marcador de progressão da doença, mas seu papel ainda tem sido controverso (Pineda et al., 2019). Em particular, foi criada a hipótese de que os linfócitos T citotóxicos (CTL) CD8 positivos para HTLV-1 específicos contra HTLV-1 desempenham um papel central no desenvolvimento de HAM/TSP. A CPV do HTLV-1, que está fortemente relacionada ao risco de desenvolver HAM/TSP, permanece relativamente estável em cada indivíduo, enquanto o HTLV-1 conduz uma forte proliferação de células T infectadas (Bangham, et al., 2015). Sabe-se que a CPV e a produção de citocinas pró-inflamatórias aumentam em pacientes com HAM/TSP e que elas desempenham um papel na patogênese da doença (Araujo \& Wedemann, 2019).

Em portadores assintomáticos, recomenda-se a medição de CPV por aparentar ser um bom preditor de doença. No entanto, estudos longitudinais mostram que, embora este marcador tenda a ser significativamente maior no início do HAM/TSP, seus valores não mudam significativamente ao longo do tempo, exceto pela flutuação não significativa no mesmo paciente (Martin, et al., 2010; Tanajura, et al., 2015).

Este estudo teve como objetivo analisar os níveis da carga proviral em portadores de HTLV-1 com diferentes condições de comprometimento neurológico. 


\section{Metodologia}

Foi realizado um estudo transversal, de natureza quantitativa (Pereira, et al., 2018), no período de março a outubro de 2017, de sujeitos com anticorpos anti-HTLV e confirmação da infecção pelo HTLV-1 por métodos de biologia molecular, atendidos no ambulatório do Núcleo de Medicina Tropical (NMT) da Universidade Federal do Pará (UFPA).

O estudo teve como locais de pesquisa o Laboratório de Biologia Molecular e Celular (LBMC), o Laboratório de Virologia (LABVIR) e o Laboratório de Clínica e Epidemiologia de Doenças Endêmicas (LCEDE) do NMT, no qual se investigou uma amostra não probabilística, intencional e convencional, composta por sujeitos advindos por demanda espontânea, encaminhados por unidades de saúde de referência, ou convidados via telefônica a partir do banco de dados do LCEDE.

Os indivíduos participaram de uma triagem com testes sorológicos e moleculares realizados no LBMC, além de receberem acompanhamento médico, avaliação neurológica e fisioterapêutica no LCEDE e terem seus dados registrados em prontuários, tais como informações quanto à identificação, procedência, dados clínicos como sinais, sintomas e achados no exame clínico e dados laboratoriais relativos aos resultados de sorologia, biologia molecular e quantificação de CPV.

A avaliação clínica dos indivíduos consistiu em um exame clínico funcional a ser realizado por uma equipe multiprofissional (neurologista e fisioterapeuta) em que, de acordo com os critérios de avaliação, os sujeitos foram divididos em três grupos por ordem decrescente de envolvimento neurológico, segundo proposta atualizada dos critérios de diagnóstico clínico para HAM/TSP (De Castro-Costa et al., 2006): Grupo 1 (Definidos para HAM/TSP), Grupo 2 (Prováveis ou Possíveis para HAM/TSP) e Grupo 3 (Sem HAM/TSP).

Em resumo, considerou-se HAM/TSP definida como uma paraparesia espástica progressiva não persistente com marcha prejudicada suficiente para ser percebida pelo paciente. Sintomas ou sinais sensoriais podem não estar presentes, mas, quando presentes, são sutis e sem um nível sensorial claro. Sinais ou sintomas urinários podem ou não estar presentes; HAM/TSP provável foi definida por uma apresentação monossintomática: espasticidade ou hiperreflexia nos membros inferiores ou sinal de Babinski isolado com ou sem sinais ou sintomas sensoriais sutis ou bexiga neurogênica somente confirmada por testes urodinâmicos. Para as denominações "definidas" e "prováveis", a equipe excluiu uma série de distúrbios que poderiam imitar a HAM/TSP; HAM/TSP possível foi definida por completa ou incompleta apresentação clínica em um ambiente onde os distúrbios que se assemelham HAM/TSP não foram excluídos. Anticorpos Anti-HTLV-1 no soro e confirmação por diagnóstico molecular foram parte de todos dos critérios acima mencionados conforme metodologia empregada no estudo de Silva et al (2018).

A detecção da CPV foi realizada no LABVIR por meio da técnica de amplificação em tempo real (Real Time PCR) de um fragmento do gene pol do HTLV-1 concomitante a hibridização do fragmento amplificado com sonda específica para o tipo viral. Como controle endógeno interno da reação, foi utilizado o gene humano da Albumina (Dehee, et al., 2002). Para classificação, a CPV foi estratificada em três níveis: baixa, valor <1.000 cópias $/ 10^{6}$ PBMC; média, acima de 1.000 a 10.000 cópias/106 PBMC; e alta, acima de 10.000 cópias/106 PBMC (Loureiro, 2008).

O programa Excel (Microsoft for Windows - 2010) foi adotado para entrada dos dados, bem como para a estatística descritiva. A análise estatística e a confecção do gráfico foram realizadas por meio do BioEstat 5.0®. Para a avaliação da normalidade dos dados de CPV, utilizou-se o teste Lilliefors a cada dois grupos (G1xG2, G1xG3 e G2xG3). Nos pares com distribuição normal foi utilizado teste T e, os com distribuição não normal, o teste Man Whitney para a análise da diferença da média/mediana de CPV entre os grupos com e sem comprometimento neurológico. O teste ANOVA (um critério), pós-teste Tukey, foi utilizado para verificar a diferença intergrupo entre as médias de CPV (p-valor $\leq 0,05)$.

Este estudo foi submetido ao Comitê de Ética em Pesquisa Envolvendo Seres Humanos (CEP) do Núcleo de Medicina Tropical - NMT/UFPA, seguindo as normas da Resolução 466/2012 do Conselho Nacional de Saúde, relativa à pesquisa em 
seres humanos, sendo aprovado sob o parecer n 1.898.095/2017.

\section{Resultados}

A amostra estudada foi constituída por 31 mulheres e 12 homens, com idade média de 51,06 anos (DP $\pm 12,94)$, variando de 22 a 81 anos. A Tabela 1 revela uma estratificação da carga proviral de HTLV nos indivíduos em três níveis: baixa, valor $<1.000$ cópias $/ 10^{6} \mathrm{PBMC}$; média, acima de 1.000 até 10.000 cópias $/ 10^{6} \mathrm{PBMC}$; e alta, acima de 10.000 cópias $/ 10^{6}$ PBMC.

Tabela 1 - Distribuição dos 43 portadores de HTLV-1 segundo o nível de carga proviral, BelémPA.

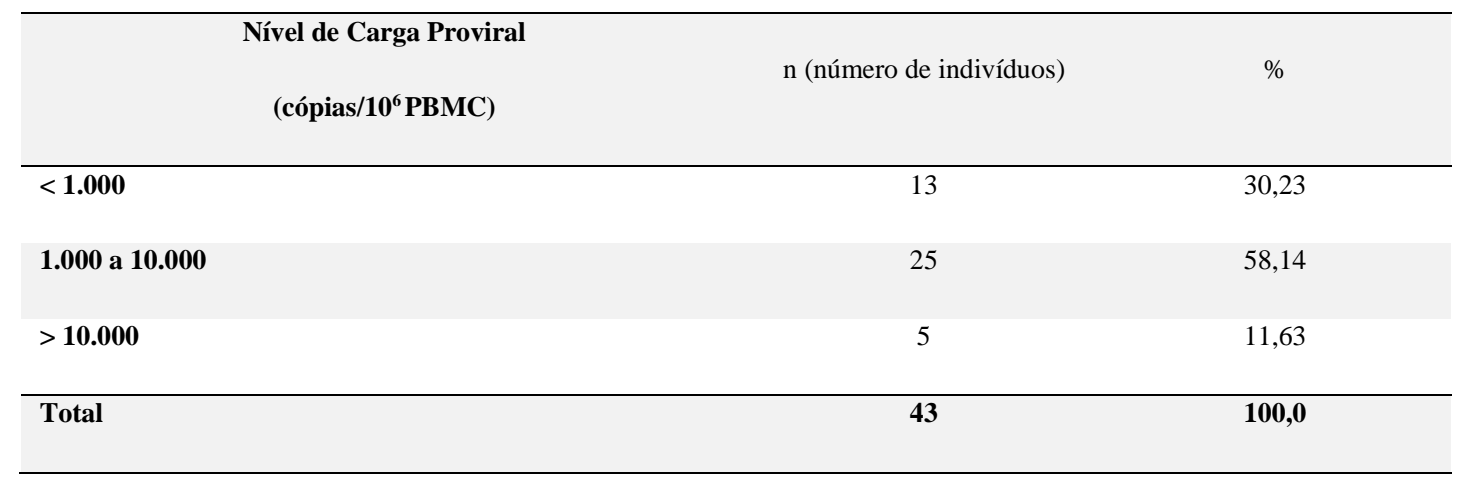

Fonte: Autores (2021).

Esses dados mostram uma frequência $(58,14 \%)$ maior de indivíduos portadores de HTLV, com nível médio de CPV entre 1.000 a 10.000 cópias $/ 10^{6}$ PBMC.

Na Tabela 2, evidenciou-se que, dentre os 43 sujeitos analisados, 27 foram considerados sem HAM/TSP do ponto de vista clínico (grupo 3), apresentando uma CPV média de 2,81 cópias $/ 10^{6}$ PBMC, com um baixo desvio-padrão e valores variando de zero a 19,00 cópias $/ 10^{6} \mathrm{PBMC}$.

Tabela 2. Medidas de tendência central e de dispersão das CPV dos 43 portadores de HTLV-1, de acordo com diferentes condições de comprometimento neurológico, Belém-PA.

\begin{tabular}{|c|c|c|c|c|c|c|c|}
\hline \multirow[t]{2}{*}{ Status clínico } & \multicolumn{7}{|c|}{$\begin{array}{r}\text { CARGA PROVIRAL } \\
\text { (cópias } / 10^{6} \mathrm{PBMC} \text { ) }\end{array}$} \\
\hline & $\mathrm{n}$ & Mediana & Média & Desvio-padrão & $\begin{array}{l}\mathrm{CV} \\
(\%)\end{array}$ & $\begin{array}{l}\text { Limite } \\
\text { Inferior }\end{array}$ & $\begin{array}{r}\text { Limite } \\
\text { Superior }\end{array}$ \\
\hline $\begin{array}{c}\text { Grupo } 1 \\
\text { Com HAM/TSP }\end{array}$ & 7 & 4,00 & 9,00 & 11,18 & 124,23 & 1,00 & 33,00 \\
\hline $\begin{array}{c}\text { Grupo } 2 \\
\text { Provável/Possível } \\
\text { para HAM/TSP }\end{array}$ & 9 & 0,00 & 1,89 & 3,92 & 207,49 & 0,00 & 12,00 \\
\hline $\begin{array}{c}\text { Grupo } 3 \\
\text { Sem HAM/TSP }\end{array}$ & 27 & 2,00 & 2,81 & 4,04 & 143,48 & 0,00 & 19,00 \\
\hline
\end{tabular}

$\mathrm{n}$ - número de indivíduos, $\mathrm{CV}$ - coeficiente de variação,

Fonte: Autores (2021)

Outras medidas importantes a serem observadas na Tabela 2, são: a baixa mediana e o alto coeficiente de variação 
(CV) de CPV do grupo de portadores com Provável/Possível para HAM/TSP (Grupo 2). Além disso, aqueles com HAM/TSP (Grupo 1) apresentaram média de CPV três vezes superior à do Grupo 3 (sem HAM/TSP).

Na Figura 1, observam-se as diferenças entre as médias de CPV entre pares dos três grupos de estudo com diferentes condições de comprometimento neurológico.

Figura 1. Análise da Diferença entre as médias de cópias $/ 10^{6}$ PBMC de CPV intergrupos de portadores de HTLV-1 com diferentes condições de comprometimento neurológico (ANOVA: um critério, ns - não significativo).

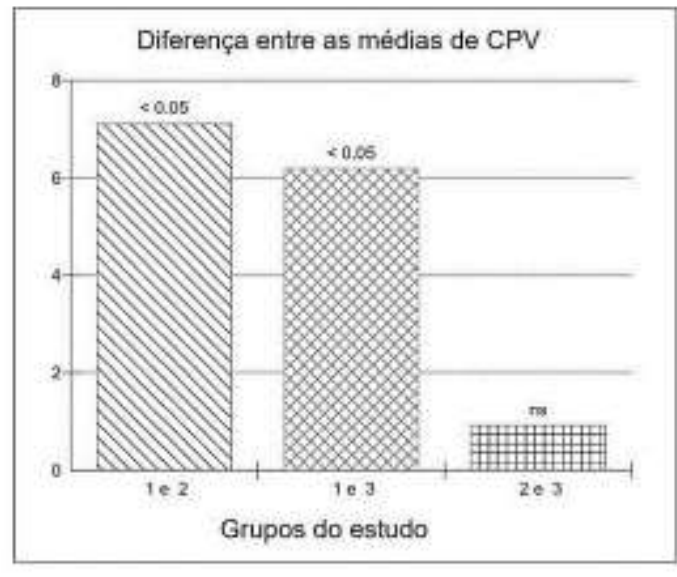

Fonte: Autores (2021).

Nesta análise da Figura 1, nota-se uma diferença significativa $(\mathrm{p}<0,05)$ entre as médias de CPV do grupo com HAM/TSP (G1) frente aos grupos com Provável/Possível HAM/TSP (G2) e sem HAM/TSP (G3).

A Tabela 3 mostra as medianas e quartis dos portadores de HTLV-1 com comprometimento neurológico associado ao vírus $(\mathrm{G} 1+\mathrm{G} 2)$ e daqueles sem comprometimento neurológico (G3).

Tabela 3 - Análise das medianas e quartis dos portadores de HTLV-1, de acordo com comprometimento neurológico, Belém-PA.

\begin{tabular}{lcc}
\hline & Número de Casos & Mediana (Q1 e Q3)* \\
\hline Com comprometimento neurológico $(\mathbf{G 1}+\mathbf{G 2})$ & 16 & $2,00(0$ e 4,75$)$ \\
Sem comprometimento neurológico $(\mathbf{G 3})$ & 27 & $2,00(0$ e 3,00$)$ \\
\hline
\end{tabular}

*Mediana de Carga Proviral (cópias/10 $10^{6}$ PMC); Q1 - Primeiro quartil (25\%); Q3 - Terceiro quartil (75\%)

** Teste de Mann-Whitney

Fonte: Autores (2021).

$\mathrm{Na}$ análise da Tabela 3, observa-se que não houve diferença significativa entre as medianas dos valores de CPV dos portadores de HTLV-1 com comprometimento neurológico associado ao vírus (G1 + G2), em relação ao grupo sem sintomas neurológicos (G3).

\section{Discussão}

A infecção pelo HTLV-1 causa ao portador várias dificuldades, desde o diagnóstico laboratorial, na identificação do paciente verdadeiramente portador da infecção, na evolução clínica, com riscos constantes de desenvolvimento de doenças associadas, até a inexistência de um tratamento que proponha a cura ou o controle da proliferação do retrovírus no organismo 
humano. Embora invisível para a sociedade, ainda não reconhecida como prioridade pelas políticas públicas e desconhecida até mesmo por muitos profissionais de saúde, a infecção pelo HTLV pode constituir um estigma para aqueles que convivem com o vírus, que os marca e os acompanham pela vida (Garcia \& Hennington, 2019).

Vários autores têm publicado sobre a importância da associação direta do nível de CPV com o desenvolvimento de doenças associadas, verificando que pacientes com HAM/TSP tem uma carga proviral maior que a de portadores assintomáticos e com ATL (Jeffery, et al., 1999; Manns, et al., 1999; Montanheiro, et al., 2005; Olavarria, et al., 2006; Loureiro, 2008; Grassi et al., 2011, Grassi, et al., 2013). Para Loureiro (2008), a detecção da CPV pela técnica da PCR em tempo real é relevante no monitoramento do número de células sanguíneas periféricas infectadas e na avaliação da propensão às enfermidades associadas ao HTLV-1.

Em uma pesquisa realizada em Kagoshima (Japão) em 1998, foi observada uma CPV cinco vezes maior em pessoas com HAM/TSP comparado a indivíduos portadores de HTLV-1 assintomáticos (Hashimoto, et al., 1998). Em estudo brasileiro mais atual, desenvolvido em São Paulo, comparou-se 45 indivíduos assintomáticos com 44 portadores de HAM/TSP e encontrou-se também uma diferença significativa entre a CPV dos dois grupos, sendo a CPV média daqueles com HAM/TSP 2,5 vezes maior que a dos assintomáticos (Montanheiro, et al., 2005). Esses resultados corroboram o encontrado no presente estudo, onde foi observada média de CPV três vezes superior naqueles com HAM/TSP (Grupo 1), quando comparada ao grupo sem HAM/TSP (Grupo 3). Entretanto, outros estudos demonstram CPV dos portadores de HAM/TSP 17 vezes maior (Manns, et al., 1999), 16 vezes maior (Nagai, et al., 1998) e 14 vezes maior (Kubota, et al., 1993; Olavarria, et al., 2006).

Segundo Batista et al. (2019), a carga proviral do HTLV-1 é um dos principais parâmetros relacionados ao desenvolvimento de doenças associadas ao HTLV-1 em adultos. No presente estudo, a CPV não se mostrou ser um indicador de condições clínicas iniciais da HAM/TSP por não apresentar diferença significativa das médias de CPV do grupo com provável HAM/TSP (G2) em relação ao grupo sem sintomas neurológicos (G3). Esses resultados foram de encontro aos achados de Haziot et al (2019), no qual se descreveu uma associação da infecção pelo HTLV-1 com uma variedade de manifestações clínicas ocorrendo em pacientes que não têm ou que não desenvolveram HAM/TSP completa ainda. Embora se tenha observado diferença significativa na média de CPV apenas no grupo com manifestações clássicas e definidas para HAM/TSP (G1) em relação aos demais (G2 e G3), sintomas neurológicos devem ser identificados precocemente devido à sua alta prevalência e interferência na qualidade de vida da pessoa que vive com o HTLV-1.

\section{Conclusão}

A medição da CPV de HTLV-1 parece auxiliar a avaliação clínica do comprometimento neurológico para a classificação dos casos clássicos de HAM/TSP, porém, nota-se não ser um indicador importante de condições clínicas iniciais da doença.

Torna-se necessário ampliar o estudo para dar mais robustez aos resultados encontrados, bem como, torna-se útil a quantificação de CPV para entender o estado clínico da HAM/TSP para um melhor acompanhamento e intervenção clínica adequada.

\section{Agradecimentos}

Os autores agradecem a todas as pessoas que vivem com HTLV-1 e que participaram desse estudo de forma voluntária. Os agradecimentos também são estendidos às agências de fomento, pelo apoio financeiro do Conselho Nacional de Desenvolvimento Científico e Tecnológico (CNPq) (MCTI / CNPq / Universal 14/2014, n ${ }^{\circ} 459352$ / 2014-8) e pelas bolsas estudantis da Coordenação de Aperfeiçoamento de Pessoal de Nível Superior (CAPES) e Universidade Federal do Pará. Essas fontes de financiamento não tiveram envolvimento na condução da pesquisa e/ou elaboração do artigo. 


\section{Referências}

Araujo, A. Q. C., \& Wedemann, D. (2019). HTLV-1 Associated Neurological Complex. What is Hidden below the Water? AIDS Reviews. $21,211-217$.

Araujo, A. Q. C. (2019) Neurologic complications of HTLV-1: a review. Revista Brasileira de Neurologia. 55(2), 5-10.

Bangham, C. R., Araujo, A., Yamano, Y., \& Taylor, G. P. (2015) HTLV-1-associated myelopathy/tropical spastic paraparesis. Nature Reviews Disease Primers. 1:15012.

Batista, E. S., Oliveira, P. D., Primo, J., Varandas, C. M. N., Nunes, A. P., Bittencourt, A.L, et al. (2019) HTLV-1 proviral load in infective dermatite associated with HTLV-1 does not increase after the development of HTLV-1-associated myelopathy/tropical spastic paraparesis and does not decrease after IDH remission. PLOS Neglected Tropical Diseases. 13(12):e0007705. https://doi.org/10.1371/journal.pntd.0007705.

Carneiro-Proietti, A. B. F., Proietti, F. A., \& Catalan-Soares, B. C. (2002) Human T cell Lymphotropic viruses (HTLV/1-2) in South America: Should It Be a Public Health Concern? Journal Biomedic Sciece. 9, 587-95.

Carod-Artal, F. J. (2009) Immunopathogenesis and treatment of the myelopathy associated to the HTLV-I virus. Reviews Neurology, 48,147-155.

Catalan-Soares, B. Carneiro-Proietti, A. B. F., \& Proietti, F. A. (2005) Heterogeneous geographic distribution of human T-cell lymphotropic viruses I and II (HTLV-I/II): serological screening prevalence rates in blood donors from large urban areas in Brazil. Cadernos de Saúde Pública, 21 (3):926-993.

Cook, L. B., Cook, M., Elemans, A. G., \& Rowan, B. (2013). Asquith HTLV-1: persistence and pathogenesis, Virology. 435, 131-140.

Da Costa, C. A., Furtado, K. C., Ferreira, L. S., Almeida, D. S., Linhares, A. C., Ishak, R., Vallinoto, A. C., Lemos, J. A., Martins, L. C., Ishikawa, E. A., Sousa, R. C., \& Sousa, M. S. (2013). Familial transmission of human T-cell lymphotrophic virus: silent dissemination of an emerging but neglected infection. PLOS Neglected Tropical Diseases. 7(6), 1-7. https://doi.org/10.1371/journal.pntd.0002272.

De Castro-Costa, C. M. et al. (2006). Proposal for diagnostic criteria of tropical spastic paraparesis/HTLV-I-associated myelopathy (TSP/HAM). AIDS Research and Human Retroviruses. 22(10), 931-5.

De Thé, G., \& Bomford, R. (1993). An HTLV-I vaccine: why, how, for whom?). AIDS Research and Human Retroviruses. 9(5):381-6.

Dehee, A., Cesaire, R., \& Desire, N., et al. (2002). Quantificação da carga proviral de HTLV-I por um ensaio de PCR em tempo real TaqMan. Journal of Virological Methods, 102: 37-51.

Edlich, R. F., Hill, L. G., \& Williams, F. M. (2003). Global epidemic of human T-cell lymphotrophic virus type-I (HTLV-I): an update. Journal of Long-Term Effects of Medical Implants. 13(2):127-40

Ferreira, L. S. C., Costa, J. H. G., Costa, C. A., Melo, M. F. C, Andrade, M. L, Martins. L. C. Ishikawa, E. A. Y., \& Sousa, M. S. (2010) Soroprevalência do vírus linfotrópico de células T humanas em comunidades ribeirinhas da região nordeste do Estado do Pará, Brasil. Revista Pan-Amazônica de Saúde, v.1, p.103-108.

Garcia, I. F. S., \& Hennington, E. A. (2019). HTLV: uma infecção estigmatizante? Caderno de Saúde Pública. 35(11), e00005419. 10.1590/0102$311 \mathrm{X} 00005419$.

Gessain, A., \& Mahieux, R. (2012). Tropical spastic paraparesis and HTLV-1 associated myelopathy: clinical, epidemiological, virological and therapeutic aspects. Revue Neurologique. Paris, 168, 3.

Grassi, M. F. R., Olavarria, V. N., Kruschewsky, R. A., Yamano, Y., Jacobson, S., Taylor, G. P., Martin, F., \& Galvão-Castro, B. (2013). Utility of HTLV proviral load quantification in diagnosis of HTLV-1-associated myelopathy requires international standardization. Journal of Clinical Virology. 58(3): 584586.

Grassi, M. R. F. G., Olavarria, V. N., Kruschewsky, R. A., Mascarenhas, R. E., Dourado, I., Correia, C. L. L., Castro-Costa, C. M., \& Galvao-Castro, B. (2011) Human T Cell Lymphotropic Virus Type 1 (HTLV-1) Proviral Load of HTLV-Associated Myelopathy/Tropical Spastic Paraparesis (HAM/TSP) Patients According to New Diagnostic Criteria of HAM/TSP. Journal of Medical Virology. 83, 1269-1274.

Hashimoto, K. et al. (1998) Quantitative in situ PCR assay of HTLV-1 infected cells in peripheral blood lymphocytes of patients with ATL, HAM/TSP and asymptomatic carriers. Journal of the Neurological Science, Amsterdam, 159, (1)67-72.

Haziot, M. E., Gascon, M. R., Assone, T., Fonseca, L. A. M., Luiz, O. D. C., Smid, J., et al. (2019) Detection of clinical and neurological signs in apparently asymptomatic HTLV-1 infected carriers: Association with high proviral load. PLOS Neglected Tropical Diseases. 13(5): e006967. https://doi.org/10.1371/journal.pntd.0006967.

Jeffery, K., et al. (1999) HLA alleles determine human T-lymphotropic virus-I (HTLV-I) proviral load and the risk of associated myelopathy. Procedings of National Academy of Sciences United States of America, Washington, 96, 3848-3853.

Kaplan, J. E., Osame, M., Kubota, H., et al. (1990) The risk of development of HTLV-I-associated myelopathy/tropical spastic paraparesis among persons infected with HTLV-I. Journal of Acquired Immune Deficiency Syndromes. 3(11): 1096-101.

Koga, Y., Iwanaga, M., Soda, M., Inokuchi, N., Sasaki, D., Hasegawa, H., Yanagihara, K., et al. (2010). Trends in HTLV-1 prevalence and incidence of adult T-cell leukemia/lymphoma in Nagasaki, Japan. Journal of Medical Virology. 82(4):668-74.

Kondo, T., Kono, H., Miyamoto, N., Yoshida, R., Toki, H., Matsumoto, I., Hara, M., et al. (1989) Age- and sex-specific cumulative rate and risk of ATLL for HTLV-I carriers. International Journal of Cancer.15, 43(6):1061-1064. 
Kubota, R., et al. (1993) Fluctuation of HTLV-I proviral DNA in peripheral blood mononuclear cells of HTLV-I-associated myelopathy. Journal of Neuroimmunology, Amsterdam, 42, 2,147-154.

Loureiro, P. (2008) Infecção pelo HTLV-1: diagnóstico e determinação da carga proviral em indivíduos assintomáticos e com enfermidades associadas em serviço de referência no Nordeste. Tese (Doutorado em Saúde Pública) - Centro de Pesquisas Aggeu Magalhães Fundação Oswaldo Cruz, Recife-PE.

Maloney, E. M., Cleghorn, F. R., Morgan, O. S., Rodgers-Johnson, P., Cranston, B., Jack, N., Blattner W. A., et al. (1998) Incidence of HTLV-I-associated myelopathy/tropical spastic paraparesis (HAM/TSP) in Jamaica and Trinidad. Journal of Acquired Immune Deficiency Syndromes. 1; 17(2):167-70.

Manns, A., Hisada, M., \& Grenade, L. L. (1999) Human T-lymphotropic virus type I infection. Lancet, London, 33 (9168): 1951-1958.

Martin, F., Fedina, A., Youshya, S., \& Taylor, G. P. (2010). A 15-year prospective longi- tudinal study of disease progression in patients with HTLV-1 associated myelopathy in the UK. Journal of Neurology, Neurosurgery and Psychiatry. 81:1336-40.

Montanheiro, P. A., et al. Human T-cell lymphotropic virus type I (HTLV-I) proviral DNA viral load among asymptomatic patients and patients with HTLV-Iassociated myelopathy/tropical spastic paraparesis. Brazilian Journal Medical Biological Research, Ribeirão Preto, $38(11), 2005$. <http://www.scielo.br/scielo.php?script=sci_arttext\&pid=S0100879X2005001100011\&lng=en\&nrm=iso〉.

Nagai, M., et al. (1998) Analysis of HTLV-I proviral load in 202 HAM/TSP patients and 243 asymptomatic HTLV-I carriers: high proviral load strongly predisposes to HAM/TSP. Journal of Neurovirology, Houndmills, 4, (6):586-593.

Olavarria, V. N. G., et al. (2006) HTLV-1 proviral load in peripheral mononuclear cell quantified in TSP/HAM patients, asymptomatic and oligosymptomatic HTLV-1 carries. In: Simpósio Internacional Sobre Htlv No Brasil, 9, 2006, Belo Horizonte, Temas livres. Uberaba: Sociedade Brasileira de Medicina Tropical, 102.

Oliveira-Filho, A. B., Araújo, A. P. S., Souza, A. P. C., Gomes, C. M., Silva-Oliveira, G. C., Martins, L. C., Fischer, B., Machado, L. F. A., Vallinoto, A. C. R., Ishak, R., Lemos, J. A. R., \& Kupek E. (2019). Human T-lymphotropic virus 1 and 2 among people who used illicit drugs in the state of Pará, northern Brazil. Scientific Reports. 14, 9(1), 14750. 10.1038/s41598-019-51383-7.

Osame, M. (1990) Review of WHO Kagoshima meeting and diagnostic guidelines for HAM/TSP. in: Blattner WA. Human Retrovirology: HTLV, New York: Raven, 90; 191-197.

Pereira, A. S., Shitsuka, D. M., Parreira, F. J., \& Shitsuka, R. (2018). Metodologia da pesquisa científica. UFSM.

Pineda, M. V., Bouzas, M. B., Remesar, M., Fridman, A., Remondegui, C., Mammana, L., et al. (2019) Relevance of HTLV-1 proviral load in asymptomatic and symptomatic patients living in endemic and non-endemic areas of Argentina. PLOS ONE 14(11): e225596. https://doi.org/10.1371/journal.pone.0225596.

Proietti, F. A., Carneiro-Proietti, A. B., Catalan-Soares, B. C., \& Murphy, E. L. (2005) Global epidemiology of HTLV-I infection and associated diseases. Oncogene. 5; 24(39):6058-68.

Schierhout, G., Mcgregor, S., Gessain, A., Einsiedel, L., Martinello, M., \& Kaldor, J. (2020). Association between HTLV-1 infection and adverse health outcomes: a systematic review and meta-analysis of epidemiological studies. Lancet. 20(1),133-143. https://doi.org/10.1016/S1473-3099(19)30402-5.

Silva, I. C., Pinheiro, B. T., Nobre, A. F. S., Coelho, J. L. P., Pereira, C. C. C., Ferreira, L. S. C., Almeida, C. P. S., Viana, M. N. S. A., Almeida, D. S., Falcão, J. R., Santos, Y. C. V., Araújo, M. W. L., Borges, M. S., Nascimento, L. D., Valentim, L. S., Casseb, J. S. R., Costa, C. A., \& Sousa, M. S. (2018). Moderada endemicidade da infecção pelo vírus linfotrópico-T humano na região metropolitana de Belém, Pará, Brasil / Moderate endemicity of the human Tlymphotropic virus infection in the metropolitan region of Belém, Pará, Brazil. Revista Brasileira de Epidemiologia. 21: e180018. http://dx.doi.org/10.1590/1980-549720180018.

Tanajura, D., Castro, N., Oliveira, P., Neto, A., Muniz, A., Carvalho, N. B., et al. (2015) Neurological manifestations in human T-Cell lymphotropic virus Type 1 (HTLV-1)-infected individuals without HTLV-1-associated myelopathy/ tropical spastic paraparesis: a longitudinal cohort study. Clinical Infectious Diseases. 61:49-56.

Vallinoto, A. C., Pontes, G. S., Muto, N. A., Lopes, I. G., Machado, L. F., Azevedo, V. N., et al. (2006). Identification of human T-cell lymphotropic virus infection in a semi isolated Afro-Brazilian quilombo located in the Marajó Island (Pará, Brazil). Memórias do Instituto Oswaldo Cruz. 101 (1):103-105.

Vallinoto, A. C., Muto, N. A., Pontes, G. S., Machado, L. F., Azevedo, V. N., Santos, S. E., et al. (2004). Serological and molecular evidence of HTLV-I infection among Japanese immigrants living in the Amazon region of Brazil. Japanese journal of infectious diseases. 57(4)156-159. 